\title{
Physicists about to hi-jack DNA?
}

\section{Unless molecular biologists are careful and energetic, they will find that the mechanical and thermo- dynamic properties of DNA have been taken over by physicists.}

Molecular biologists, if pressed, will readily agree that more should be done to throw light on the properties of naturally occurring macromolecules in the molecular environment in which they ordinarily exercise their biological activity, usually that of water. More than that, much is being done, experimentally and otherwise. X-ray diffraction measurements of, say, crystalline proteins are not always the abstract studies they may seem, given the large water content of many specimens. The application of nuclear magnetic resonance techniques to enzyme molecules may indirectly say much about water interactions. The abiding obstacle, in these connections and others, is that the important molecules are so often complicated in their own right, even without the extra complication of their external interactions. People sigh for simpler systems.

That is where physics may have the edge. There is probably a great deal in the opinion that biologists are reconciled to having to tackle the problems with which nature confronts them, but that physicists are free to pick and choose, tackling only the problems they can reasonably hope to solve. Put starkly, like that, the distinction is obviously a simplification. (Sir Peter Medawar's The Art of the Soluble is, in any case, a biologist's book.) But the caricature does seem to illustrate a difference of style, which is in turn nicely illustrated by a neat analysis of the properties of DNA molecules in solution by L.L.Van Zandt of Purdue University appearing in Physical Review Letters $(57,2085 ; 1986)$.

The starting point for Van Zandt's analysis is an account which appeared two years ago (Edwards, G.S., Davis, C.C., Saffer, J.D. \& Swicord, M.L. Phys. Rev. Lett. 53, 1284; 1984) of the absorption of microwaves in solutions of DNA. Part of the motivation of that work was the belief that measurements might throw light on suggestions that microwave absorption can cause biological damage even when the amounts of energy involved are very small, perhaps because of some resonance between the microwave frequency and a vibrational frequency of some vital and susceptible molecule.

But how could such a molecule, say a DNA molecule, even if characterized by well-defined frequencies of vibration, accumulate substantial amounts of energy in these vibrational modes while surrounded by water molecules whose effect would be that vibrations of any kind would be efficiently damped? Edwards and his colleagues were sustained by their earlier observation of a dramatic increase in the gross absorption of microwaves by solutions of DNA from Escherichia coli in the presence of the endonuclease DNase I. They inferred that the continuing degradation of long molecules would assure a constant supply of shorter molecules capable of vibrating resonantly with external microwaves.

So why not test this possibility by using the techniques of genetic manipulation to make pieces of DNA whose size is well defined? Edwards and colleagues did just this and made two remarkable discoveries. First, well-defined DNA molecules, whether circular or linear, do indeed show resonance absorption of microwaves in the frequency range from 1 to $10 \mathrm{GHz}$. Second, the absorption peaks are surprisingly sharp, suggesting ineffective damping by the surrounding medium.

Why that should be is for Van Zandt to say, but the numbers are interesting in themselves. The measured damping implies that the relaxation time, that needed for a vibrational impulse to be dissipated, is of the order of 300 picoseconds. But the velocity of sound in DNA is within 15 per cent of $2 \mathrm{~km} \mathrm{~s}^{-1}$. One consequence is that vibrational excitations can travel only about 1,500 base pairs along a linear DNA molecule before being dissipated, which in turn implies that coherent vibrations over much greater distances will not easily happen. That is why Edwards et al. found that their resonance frequencies were usually harmonics of a fundamental.

Van Zandt has had the benefit of an even more striking demonstration of anomalous resonance in DNA (by the Edwards group) based on measurements of DNA molecules with 5,480 base pairs derived from a circular plasmid in which supercoiling has been relieved by the use of a topoisomerase. The remarkable feature of the measurements is that the strength of the absorption (four peaks between 3 and $9 \mathrm{GHz}$ ) increases with increasing frequency, the opposite of that expected of an efficiently damped system.

The explanation can be made simple enough to suit any physicist. First, the vibrations responsible for the resonances are simple organ-pipe vibrations - standing compressional waves set up around the circular channels of the plasmids. A few simple properties of the solvent, water, are relevant. Energy dissipation in pure water arises because of the stretching and distortion of hydrogen bonds, but because these form and reform in times of the order of $10^{-11}$ seconds, they should be measurable only at frequencies of $50 \mathrm{GHz}$ and above. Van Zandt's question is how circular DNA can so profoundly modify this behaviour.

The molecular model is simple. Suppose that there is a single layer of water molecules more tightly bound to the DNA than to the bulk of the solvent, so that it will move with the molecule if that should vibrate, but at the same time be better adapted than DNA to sense and be influenced by the molecular motions in bulk liquid. Van Zandt constructs the crudest of all possible mechanical models to represent this state of affairs. Take two masses, one representing the mass of DNA (per unit length) and the other the mass of the bound water layer (again per unit length) and imagine them connected by an elastic spring. Let the second mass (the bound layer of water molecules) be damped, and calculate the absorption of the system as a function of microwave frequency.

Sceptics will say that even this simple system has enough adjustable constants to fit pretty well any measured curve. Van Zandt plays fair by using real values (the mass of DNA and the bulk viscosity of water) to fit as many of them as possible. Even the allowable frequencies of vibration are taken from measured velocities of sound in dry DNA (and the condition that there must be an integral number of wavelengths around a single circle of DNA). The result is a remarkably good fit of the position of the four absorption peaks, but the measured peaks are much broader than predicted.

Where this will lead is anybody's guess. Van Zandt promises a more detailed account of the structure of the solvent. The discrepancy between the width of the measured and predicted peaks cannot be made to go away by adjusting (and increasing) the damping constants in the model, for that has the effect of changing the relative strength of the peaks. Van Zandt thinks that even a circular plasmid whose supercoiling has been relieved may have some residual tertiary structure, but there are others who will see the difference as an opportunity to learn more about the solvent interactions. How soon will it be before molecular biology laboratories equip themselves with a klystron and some waveguide?
John Maddox 